\title{
Tachypnea detected by an acoustic respiratory rate monitor is a predictor of the occurence of oxygen desaturation
}

\author{
Mary SAAD, M.D., Jean-Louis BOURGAIN, M.D., Valérie BILLARD, M.D. \\ Institut de Cancérologie Gustave Roussy
}

\section{Introduction}

Patients undergoing neck surgery are at a higher risk of cardiac and respiratory complications (1). The first sign of respiratory compromise detected by postoperative continuous monitoring is blood oxygen desaturation. The non-invasive monitoring of respiratory rate (RR) by an acoustic monitor in the postoperative period has been shown to be as effective and better tolerated than capnometry in the monitoring of RR $(2,3)$. The purpose of this study is to evaluate the incidence of postoperative desaturation episodes in neck surgery patients and the correlation between a change in RR detected by the acoustic monitor and the occurrence of desaturation.

\begin{tabular}{|c|c|c|c|}
\hline \multirow[t]{2}{*}{ Respiratory Rate } & \multicolumn{2}{|c|}{$\begin{array}{l}\text { Probability of oxygen } \\
\text { desaturation } 10 \text { minutes later }\end{array}$} & \multirow[t]{2}{*}{ Odds ratio $(95 \% \mathrm{Cl})$} \\
\hline & yes & no & \\
\hline Less than $8 / \mathrm{min}$ & $3,7 \%$ & $5,2 \%$ & $0,7(0,6-0,8)$ \\
\hline More than $18 /$ min & $15,5 \%$ & $3,3 \%$ & $5,3(5,1-5,4)$ \\
\hline
\end{tabular}

\section{Methods}

Fifteen patients who underwent neck surgery (laser surgery of the vocal cords, thyroidectomy, neck dissection) without tracheostomy had, during the first postoperative night, continuous monitoring of pulse oxygen saturation (SpO2) and respiratory rate (RR) with an adhesive bioacoustic sensor applied to the neck and connected to a monitor (Radical 7 Masimo, Irvine, CA). RR and $\mathrm{SpO} 2$ were recorded every 2 seconds. Retrospectively, the occurence and duration of desaturation ( $\mathrm{SpO} 2<92 \%$ ) episodes were calculated for each patient. Respiratory rate was recorded at the time of desaturation, 5 minutes earlier and 10 minutes earlier. The statistical analysis was performed using SPSS software, version 21.

\section{Results}

A total of 416,137 data entries were analyzed with a monitoring median time of 15 hours. Desaturation occured during 5\% of the total monitoring period. $\mathrm{SpO} 2$ was significantly correlated to $\mathrm{RR}$ at the time of the measurement, 5 minutes earlier and 10 minutes earlier $(r=-0123, P<0.001 ; r=-0137, P<0.001$ and $r=$ $-0151, P<0.001$, respectively). Respiratory rate cutoff value for predicting the occurrence of desaturation 10 minutes later was determined by establishing a ROC curve and corresponds to $18 \mathrm{bpm}$ with a sensitivity of $0.447(95 \% \mathrm{Cl} 0.445,0.449)$, a specificity of $0.869(95 \% 0.868,0.870)$ and an area under the curve of $0.677(95 \% \mathrm{Cl} 0.673,0.682), \quad P<0.001$. With a definition of tachypnea as a $\mathrm{RR}>18 \mathrm{bpm}, 45 \%$ of desaturation entries were preceded by tachypnea vs $13 \%$ of normal saturation, $\mathrm{P}<0.001$ (Figure 1). The occurence of tachypnea was associated with a 5.3 fold increase in the risk of oxygen desaturation 10 minutes later $(95 \% \mathrm{Cl} 5,1-5,4)$

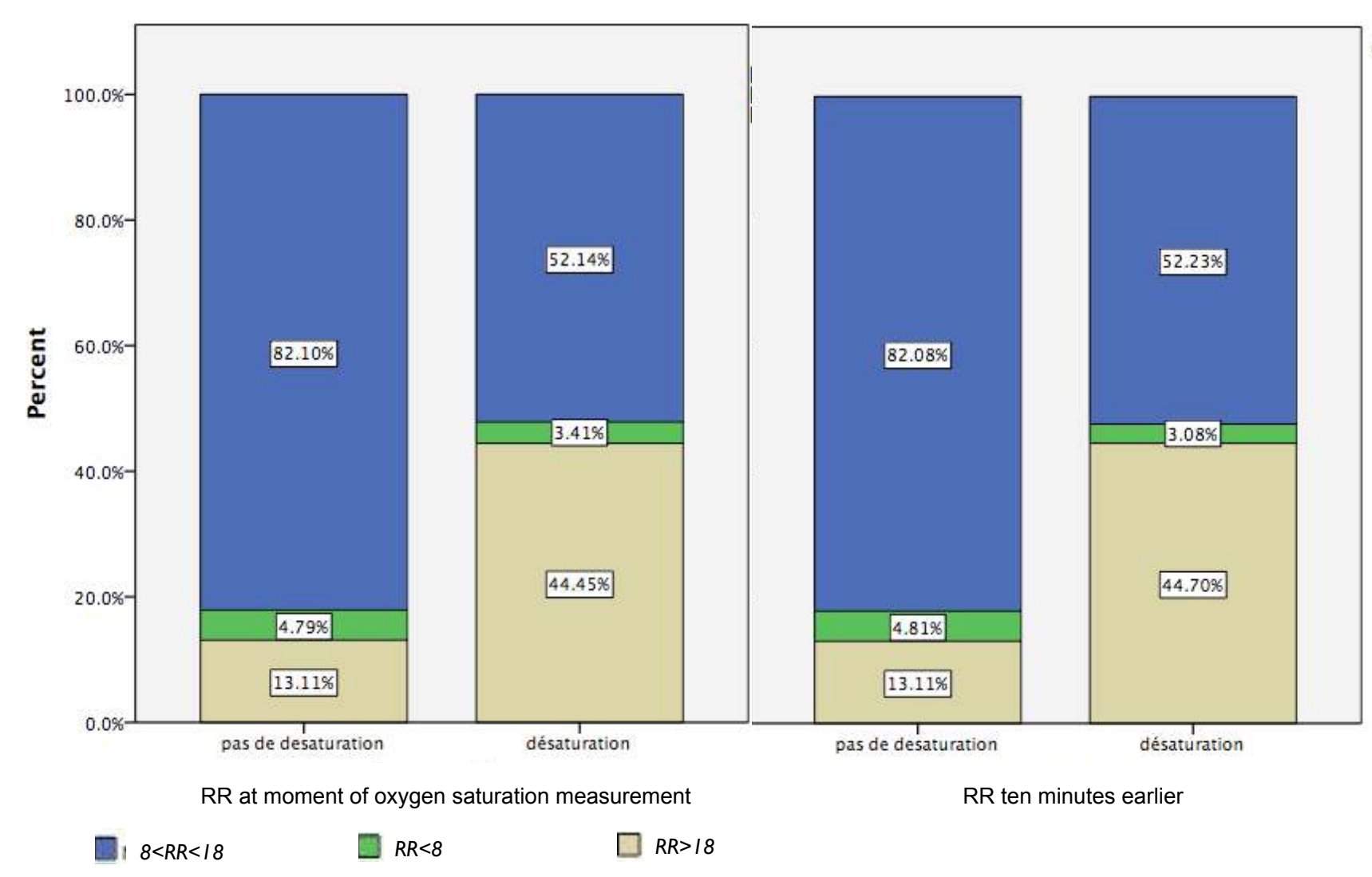

\section{Conclusion}

In patients who underwent neck surgery, an increase in respiratory rate detected by an acoustic monitor during the postoperative period is a predictor of the occurrence of desaturation ten minutes later. 